\title{
Mei R. Fu: mHealth facilitates real-time detection and prediction of lymphedema
}

Received: 30 October 2018; Accepted: 31 October 2018; Published: 09 November 2018.

doi: $10.21037 /$ mhealth.2018.11.01

View this article at: http://dx.doi.org/10.21037/mhealth.2018.11.01

\section{Editor's note}

Lymphedema, by definition swelling in extremities resulting from impaired flow of the lymphatic system, is a common leftover problem for patients who have undergone cancer treatment. In the US, it most commonly occurs in women after receiving breast cancer surgery, particularly when followed by radiation treatment. While physiotherapy and compression therapy are frequently employed to reduce the swelling and discomfort of lymphedema patients, there is still no known cure for this medical condition. Worse still, the short of systematic and effective evaluations and interventions of symptoms after cancer treatment poses a roadblock for early detection of lymphedema.

As a nurse scientist, Prof. Mei R. Fu from New York University (NYU) Rory Meyers College of Nursing, NYU witnessed how patients suffered from lymphedema, and felt the strong need to help them relieve their symptoms. Having spent years of efforts in the study of cancer-related symptoms and chronic illnesses with a special focus on lymphedema, Prof. Fu has been incorporating qualitative and quantitative methods, genomic and biomarker approaches, cutting edge measurement technology and innovative behavioral interventions in her research. With the emergence of $m$ Health, Prof. Fu is seeking a way where real-time detection and prediction of chronic illness due to cancer treatment such as lymphedema can be made feasible. The following interview will delve into the research she has been working on particularly in this area.

\section{Expert introduction}

Prof. Mei R. Fu (PhD, RN, FAAN) is an internationally and nationally well-known nurse scientist and outstanding researcher and educator (Figure 1). She is a Tenured Associate Professor of Nursing at NYU Rory Meyers College of Nursing, NYU. Prof. Fu is also a Fellow of American Academy of Nursing, the highest professional honor recognizing outstanding contributions, achievements,

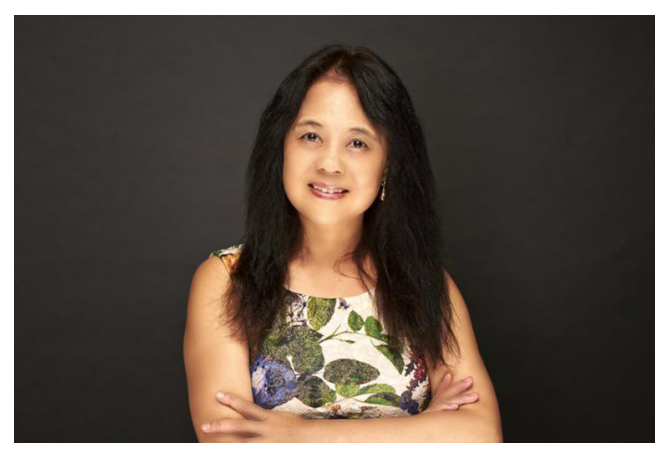

Figure 1 Prof. Mei R. Fu.

and leadership in the nursing field; a Fellow of Geriatrics at the Hartford Institute of Geriatrics, the highest honor for Prof. Fu's expert knowledge contributed to the field of geriatrics; and a Fellow of New York Academy of Medicine, recognition of the highest levels of achievement and leadership in the fields of urban health, science, social work, nursing, education, law, medicine and research. She was inducted into the Sigma Theta Tau International (STTI) International Nurse Researcher Hall of Fame in 2017 for her lifetime achievements in and contributions to research and for mentoring future nurse researchers.

Prof. Fu's scientific focus has been on cancer-related symptoms and management of chronic illnesses. Her research incorporates qualitative and quantitative methods, genomic and biomarker approaches and cutting-edge measurement technology as well as innovative behavioral interventions. Her research has been supported by National Institute of Health (NIH), National Science Foundation, the Oncology Nursing Society (ONS), the Hartford Institute of Geriatric Nursing, the Avon Foundation, the Vital Fund, Judges and Lawyers for Breast Cancer Alert, Pfizer Independent Learning and Change grant. She has over 100 high quality publications in peer-reviewed journals, book chapters, and professional publications. She has over 200 invited or peer-reviewed keynote and 
podium presentations at international, national, regional, and local conferences. The significance of her research has been recognized with funding in highly competitive grant proposals. Her creative research has been recognized with prestigious research awards from ONS and International Lymphology Association. In addition, she was awarded the best article award by ONS, outstanding journal article by Fournal of Nursing Diagnosis, and Eminent Scientist of the Year 2003 by International Research Promotion Council.

\section{Interview}

mHealth: Having been studying cancer-related symptoms and chronic illnesses for years, what do you think are the critical issues facing these areas right now?

Prof. Fu: Symptoms are subjectively perceived indicators of abnormal biological or physiological changes that may or may not be observed objectively. Being diagnosed with and treated for cancer is a devastating experience. What's more frustrating is the unexpected suffering of symptoms from secondary chronic illnesses due to cancer treatment, such as lymphedema (i.e., an abnormal lymph fluid accumulation) or neuropathic pain. Lack of accessible, systematic and sustained ongoing evaluations of symptoms after cancer treatment and effective interventions remains critical issues faced by patients and healthcare providers. For example, symptoms may indicate an early stage of lymphedema that constitutes only minimal changes in objective measures of limb volume increase. Thus, early detection of symptoms is essential for early detection of lymphedema. Despite its value in detecting early-stage lymphedema, the use of symptom assessment in clinical settings is still absent. In addition, more research is needed to develop effective and accessible interventions for cancer-related symptoms. Technology such as mHealth is promising to address such critical issues in the area of symptoms and chronic illness.

\section{mHealth: How did you become involved in this line of research?}

Prof. Fu: My program of research on lymphedema symptom science grew out of my passion and desire to understand how patients manage lymphedema in their daily lives during my graduate study. Being a nurse who witnessed patients' daily suffering from lymphedema, I felt it imperative to be able to help patients to relieve their symptoms. Starting with qualitative inquiry to understand patients' symptom experience supported by NIH (F31 NR07851) and NYU Research Challenge Fund, I completed three descriptive phenomenological studies as the principal investigator (PI) to investigate the phenomenon of managing lymphedema in different ethnic groups, including white, Chinese American and African American breast cancer survivors. The publications based on these three qualitative research studies using a descriptive phenomenology method provided important evidence: (I) breast cancer survivors were distressed by the fact that no or limited education was given to them about lymphedema; (II) lymphedema symptom experience was described by breast cancer survivors as living with "a plethora of perpetual discomfort", which underscores the importance of lymphedema symptoms as an important patient-centered outcome; and (III) feasible self-care behaviors that were easily to integrate into daily routine were central to lymphedema management in breast cancer survivors' daily lives. This work provided strong evidence that lymphedema symptoms and their psychosocial impact should be important patient-centered clinical outcomes. In addition, feasible self-management behaviors to promote lymph flow are key to reduce the risk and management of lymphedema. By providing evidence from patients' perspectives, this body of work has changed the direction of research and clinical care on patient-centered outcomes and patient education on lymphedema risk reduction and will continue inform effective ways of performing symptom assessment and implementing self-management of lymphedema symptoms.

\section{mHealth: With the emergence of mHealth, how does it facilitate bealth care in detecting or predicting chronic illness?}

Prof. Fu: The ubiquitous availability of internet enables mHealth to play an important role in facilitating realtime detecting and predicting chronic illness due to cancer treatment such as lymphedema. First, a web-based selfassessment tool for a systematic and sustained ongoing evaluations of symptoms of lymphedema will enable ubiquitous assessment by the patients without clinical visit and large data collection beyond geographic boundaries. Such mHealth infrastructure provides necessary foundation to embed machine learning algorithm that allows to infer the patient's lymphedema risk based on the self-reported symptoms, personal and clinical features, and encourage patients at risk to visit the doctor for a formal diagnosis or 
treatment. This will reduce the cost and likelihood of early detection and intervention. The developed self-assessment tool can also be used at the physician's office, as a decision support tool for both the patient and healthcare providers. Should the developed machine learning algorithm be validated to achieve sufficiently high sensitivity and specificity in identifying lymphedema, patients do not need to go through more costly quantitative measurements at the physician's office.

mHealth: In a recent study, your team examined the use of machine learning for the detection of lymphedema among breast cancer survivors. What are the major findings of this study? What insights can it bring to guide future studies in similar areas?

Prof. Fu: In this study, we used a web-based tool to collect information from 355 women who had undergone treatment for breast cancer. In addition to sharing demographic and clinical information, including whether they had been diagnosed with lymphedema, participants were asked whether they were currently experiencing any of the more than 20 different lymphedema symptoms. We compared five different classification algorithms of machine learning: Decision Tree of C4.5, Decision Tree of C5.0, gradient boosting model, artificial neural network, and support vector machine. We also compared the algorithms with a conventional statistical approach, Youden's method, which determines the optimal threshold for the symptom count based on the receiver operating curve. We found that all five machine learning approaches outperformed the standard statistical approach, and the artificial neural network achieved the best performance for detecting lymphedema. The artificial neural network was 93.75 percent accurate, correctly classifying patients to have true lymphedema cases or non-lymphedema cases based on the symptoms reported. Thus, a well-trained artificial neural network classifier using real-time symptom reports can provide highly accurate detection of lymphedema. Such detection accuracy is significantly higher than that achievable by current and often used clinical methods, such as healthcare providers' observation of swelling. Symptoms assessment and management is the major focus for many chronic illnesses, such as cancer, chronic heart failure, HIV, or even chronic wound care. Our study provided the proof of the concept for future research in the area of symptom assessment and management as well as chronic illnesses using machine learning approach.
mHealth: Many powerful classification algorithms have been developed in the machine learning community, such as decision tree, Nä̈ve Bayes, artificial neural network $(A N N)$, and support vector machine (SVM). What do you think is the most promising classifier?

Prof. Fu: Machine learning has proven to be a powerful tool for the detection or prediction of various medical conditions and demonstrated improved accuracy over conventional statistical or expert-based systems. Machine learning is particularly beneficial when there are many relevant features and these features are not independent. This is exactly the case of lymphedema symptoms. Effective machine learning tools can discover the hidden association between a given disease (i.e., lymphedema) and the relevant symptoms, personal and clinical features, which is hard to identify using classical statistical methods. In our study, we compared five different machine learning approaches and found that the artificial neural network is best for our research. So, I would say depending on the phenomenon under investigation, a given machine learning approach might be the best fit after comparing several approaches.

\section{mHealth: Would you introduce us to a recent $\mathrm{NIH}$-funded research project that you are involved in?}

Prof. Fu: Currently, I am the Multiple PI with Dr. Yao Wang (Contact Multiple PI, Professor of NYU Tandon School of Engineering) for a three-year, technology innovation research award from the National Cancer Institute as part of a joint program on National Science Foundation Smart and Connected Health (1R01CA214085-01). The first primary goal of this project is to use machine learning to understand the association between symptoms and other relevant personal and clinical factors, and the presence of lymphedema, and develop a web-based self-assessment platform that enables patients to assess their risk for lymphedema from anywhere. This platform will provide a decision support tool for both the clinician and the patient, to facilitate patient-centered, evidence-based intervention decision. The second goal is to develop a Kinect-enhanced intervention training system, which can provide instant audio-visual feedback to the patient performing the therapeutic lymphatic exercises. The feedback will enable the patient to follow the prescribed hand/shoulder/arm movement more precisely, thereby making the intervention more effective. The R01 grant has allowed us to develop a precision assessment of lymphedema risk from patient self- 
reported symptoms through machine learning. We also have developed a Kinect-enhanced intervention training system, which can track patients' movement and provide instant audio-visual feedback to the patient, to enable the patient to follow the prescribed movement more accurately, thereby making self-care intervention more effective. The innovation of precision risk prediction and Kinect-enhanced intervention will be hosted in The-Optimal-Lymph-Flow mHealth system (TOLF).

\section{mHealth: Did you come across any bottleneck in your career/research? What have been driving you to overcome these challenges and move forward?}

Prof. Fu: My passion for lymphedema research has been sustained by my witnesses of the longsuffering of patients and the positive impact of my research on patient's quality of life. It is the desire of achieving maximized effectiveness of health care for patients that has motivated me to persistently explore innovative approaches to help patients. In my early qualitative research, I was moved by patients who were longing for the understanding of their suffering from healthcare providers and wishing more research could be done. Early in my career, the great challenge that I faced was that we needed to understand the biological pathway of lymphedema symptomology in order to advance the science of lymphedema symptoms. Supported by NIH (NINR Project\# 1R21NR012288-01A, NIMHD Project\# P60 MD000538-03) and ONS, as the PI, I and my investigative team have accomplished a muchneeded prospective investigation to discover the biological pathway of lymphedema symptomology. We documented that lymphedema symptoms are strongly associated with increased limb volume; symptoms alone can accurately detect lymphedema as well as evidence for patterns of obesity and lymph fluid level. This research has provided the evidence that a diagnostic cutoff of three symptoms discriminated breast cancer survivors with lymphedema from healthy women and a diagnostic cutoff of nine symptoms discriminated at-risk survivors and survivors with lymphedema. We also discovered that lymphedema symptoms do have an inflammatory biological mechanism as evidenced by significant relationships with several inflammatory genes. This important research provides the foundation for precision assessment of heterogeneity of the lymphedema symptom phenotype and understanding the biological mechanism of each phenotype through the exploration of inherited genetic susceptibility which is essential for finding a cure. Further exploration of investigative interventions in the context of genotype and gene expressions would further advance our understanding of heterogeneity of lymphedema symptom phenotype and make additional contributions to precision health care.

Given that the internet grants the nearly universal access to web-based programs, a technologically-driven delivery model for health care is the ultimate goal to provide universal access to health care. I and my team also faced the challenge to utilize web-and-mobilebased digital technology to deliver our intervention to help patients relieve their lymphedema symptoms and reduce their risk of lymphedema. To be able to garner the talents across disciplines at NYU, I endlessly reached out and collaborated with faculty from NYU School of Medicine (surgery, population health, pathology, and biostatistics), NYU Rusk Rehabilitation, NYU Tandon School of Engineering, NYU and the NYU Asian Center (CSAAH). Under my leadership, my team developed and pilot tested the web-based The-Optimal-Lymph-Flow mHealth system for lymphedema symptom assessment and management. The-Optimal-Lymph-Flow mHealth system is a multi-language (English, Spanish and Chinese), patient-centered behavioral therapy focusing on behavioral strategies to lessen the symptom burden by promoting lymph flow and minimize inflammation. The-Optimal-Lymph-Flow digital therapy includes information about chronic pain, lymphedema, diagnosis and measurement of lymphedema, lymphatic system, risk of lymphedema, digital therapeutic lymphatic exercises to promote lymph flow, and self-management. Patients can access the digital therapy using computer, iPhone, iPad, or other smart phones. Currently, we just completed a Randomized Clinical Trial (RCT) supported by a Pfizer Individual Learning and Change Grant (13371953 TheOptimal Lymph-Flow ${ }^{\mathrm{TM}}$ ) on digital therapy for chronic pain and lymphedema symptoms. Patients in the clinical trial loved The-Optimal-Lymph-Flow digital therapy, especially the use of avatar technology that demonstrates the complicated lymphatic system and illustrates the physiological functions of and detailed step-by-step instructions for each therapeutic exercise. Conducting meaningful research that improves patients' quality of life and reduce patients' risk for lymphedema has been the goal of my research. The emails and verbal expressions of gratefulness from patients have been the driving force for me to move forward until we find a cure for lymphedema and pain. 
mHealth: As the Editorial Board Member of mHealth, do you have any words for our readers/peers in the same field?

Prof. Fu: More and more technology enhanced program including artificial intelligence will be incorporated in patient care. We need to always remember that patient is the center of all these technology developments. It is important to involve our patients from the very beginning in the development of any technology enhanced patient care program. Finally, we should always remember that human connection is central for patient care and human connection is always essential for finding a cure for illnesses.

doi: $10.21037 /$ mhealth.2018.11.01

Cite this article as: Li B. Mei R. Fu: mHealth facilitates real-time detection and prediction of lymphedema. mHealth 2018;4:53.

\section{Acknowledgements}

We would like to express our sincerest gratitude to Prof. Mei R. Fu for sharing her insights and opinions with us.

\section{Footnote}

Conflicts of Interest: The author has no conflicts of interest to declare.

(Science Editor: Brad Li, mHealth, editor@themhealth.org) 